\title{
Pathogenesis of Neuropsychiatric Syndromes of Systemic Lupus Erythematosus
}

\author{
Taku Yoshio1* ${ }^{*}$ Hiroshi Okamoto ${ }^{2}$ \\ ${ }^{1}$ Division of Rheumatology and Clinical Immunology, School of Medicine, Jichi Medical University, Tochigi, Japan \\ ${ }^{2}$ Minami-Otsuka Institute of Technology, Minami-Otsuka Clinic, Tokyo, Japan \\ Email: ${ }^{*}$ takuyosh@jichi.ac.jp
}

Received 10 April 2015; accepted 10 May 2015; published 13 May 2015

Copyright (C) 2015 by authors and Scientific Research Publishing Inc.

This work is licensed under the Creative Commons Attribution International License (CC BY).

http://creativecommons.org/licenses/by/4.0/

(c) (i) Open Access

\begin{abstract}
The pathogenesis of neuropsychiatric syndromes of systemic lupus erythematosus (NPSLE) is multifactorial and can involve various inflammatory cytokines, autoantibodies such as anti-neuronal antibodies, anti-ribosomal $P$ antibodies, anti-NR2 glutamate receptor binding antibodies, anti-Sm antibodies, anti-U1-RNP antibodies and anti-phospholipid antibodies, and immune complexes (IC). Disruption of the blood-brain barrier (BBB) is integral to the neuropathology of SLE. Recently the possibility has been reported that aforementioned autoantibodies in the circulation may be strongly associated with disruption of the BBB. Each of these mechanisms might contribute to the pathogenesis of focal NPSLE (for example, cerebrovascular disease, movement disorders, myelopathy, seizures and cranial neuropathy) or diffuse NPSLE (for example, acute confusional state, psychosis and cognitive dysfunction) to varying degrees. In this review we focus on how the aforementioned autoantibodies, the BBB, IC and cytokines as well as chemokines are associated with the appearance of NPSLE.
\end{abstract}

\section{Keywords}

Neuropsychiatric Systemic Lupus Erythematosus, Anti-Ribosomal P Antibodies, Anti-NR2 Glutamate Receptor Binding Antibodies, Interleukin-6, Granulocyte-Colony Stimulating Factor, The Blood-Brain Barrier

\section{Introduction}

Neuropsychiatric manifestations are increasingly recognized in patients with systemic lupus erythematosus *Corresponding author.

How to cite this paper: Yoshio, T. and Okamoto, H. (2015) Pathogenesis of Neuropsychiatric Syndromes of Systemic Lupus Erythematosus. Open Journal of Rheumatology and Autoimmune Diseases, 5, 46-56. 
(SLE). These manifestations encompass a wide variety of neurologic and psychiatric features, and account for considerable morbidity and mortality in these patients. Neuropsychiatric SLE (NPSLE) involves both the central nervous system (CNS) and the peripheral nervous system, and ranges from subtle abnormalities of cognitive dysfunction and anxiety to overt manifestations, such as stroke, seizures and psychosis.

NPSLE, which involves only the CNS (central NPSLE), has been reported to occur in 14\% - 75\% of SLE patients. These large differences among the reports might be owing to differences in study design, ethnic and other demographic characteristics of cohorts, follow-up durations, and attribution of neuropsychiatric events to SLE.

In Japan our three-year prospective study showed that a large proportion (50\%) of central NPSLE events occurred at disease onset or within the first 1 year after SLE diagnosis, and 25\% of patients at disease onset or within the first 1 year after SLE diagnosis had central NPSLE events (unpublished data). In addition, $42 \%$ of central NPSLE events in the patients over the first 1 year after SLE diagnosis were reoccurrence of NPSLE (unpublished data). Furthermore our three-year prospective study predicted that of all 53,000 SLE patients in Japan about 1000 patients have central NPSLE every year (a year occurrence prevalence of central NPSLE in Japan is $1.9 \%$ ) (unpublished data).

Three major SLE-related risk factors have been consistently associated with NPSLE. The first risk factor is the increased SLE disease activity, for example the increased SLEDAI scores, or major organ involvements other than CNS, or serological activity, for example high titers of anti-dsDNA antibodies and low serum complement levels. The second risk factor is the treatment with high doses of corticosteroids because this treatment might decrease the brain blood perfusion, resulting in the deterioration of diffuse neuropsychiatric symptoms. The third risk factor is the previous or concurrent major NPSLE events, particularly stroke, seizure disorders and diffuse neuropsychiatric manifestations, which predict similar, future neuropsychiatric events.

Although the relations between the occurrence and prognosis of NPSLE and genetic or environmental factors are investigated, the strongly significant relations have not been identified.

The pathogenesis of NPSLE is multifactorial and can involve various inflammatory cytokines, autoantibodies such as anti-neuronal antibodies, anti-ribosomal $\mathrm{P}$ antibodies (anti-P), anti-NR2 glutamate receptor binding antibodies (anti-NR2), anti-Sm antibodies (anti-Sm), anti-U1-RNP antibodies (anti-RNP) and anti-phospholipid antibodies (anti-PL), and immune complexes (IC). The most common microscopic brain finding in SLE seems to be microvasculopathy which though not specific, may be due to complement activation and anti-PL [1]. These NPSLE-associated autoantibodies culminate in neuronal dysfunction and vasculopathy, intrathecal production of inflammatory cytokines, and accelerated atherosclerosis. Post mortem histopathologic studies in patients with SLE have demonstrated an array of pathologies including multifocal microinfarcts, gross infarcts, hemorrhage, cortical atrophy, ischemic demyelination, and patchy multiple sclerosis-like demyelination [2]. Disruption of the blood-brain barrier (BBB) is integral to the neuropathology of SLE [3]. Recently the possibility has been reported that aforementioned autoantibodies in the circulation may be strongly associated with disruption of the BBB. Each of these mechanisms might contribute to the pathogenesis of focal NPSLE (for example, cerebrovascular disease, movement disorders, myelopathy, seizures and cranial neuropathy) or diffuse NPSLE (for example, acute confusional state [ACS], psychosis and cognitive dysfunction) to varying degrees.

The treatment with corticosteroids is widely used as the first choice in the management of NPSLE. Glucocorticoids are used in intravenously (usually 1 gram daily for 3 days as methylprednisolone pulse therapy, followed by daily high-dose oral prednisolone [1 - $2 \mathrm{mg}$ per kg daily]) for acute and severe flares. Additional immunosuppressive agents (usually intravenous cyclophosphamide 500 - $1000 \mathrm{mg}$ once a month) are often required until neuropsychiatric symptoms subside because many NPSLE patients do not respond to the treatment with corticosteroids. In patients with severe NPSLE refractory to immunosuppressive therapy, the use of plasma exchange, intravenous immunoglobulin and rituximab (a monoclonal antibody against CD20) has been reported in uncontrolled studies, with varying rates of success. Rituximab might be the most effective therapy for severe refractory NPSLE because a considerable number of observational studies and case reports have demonstrated encouraging early remission with rituximab in cases of severe refractory NPSLE. The randomized controlled trial study of rituximab against severe refractory NPSLE is requested to elucidate the effect of rituximab.

In this review we focus on how the aforementioned autoantibodies, the BBB, IC and cytokines as well aschemokines are associated with the appearance of NPSLE.

\section{Anti-Phospholipid Antibodies}

a-PL include anti-cardiolipin antibodies, anti- $\beta 2$ glycoprotein I antibodies and lupus anticoagulant [4]. Studies 
have demonstrated a-PL-mediated direct neuronal injury in the absence of ischemia [5]-[8]. Persistently elevated anti-cardiolipin antibodies are associated with greater cognitive impairment [9] [10]. In a cohort of 1000 patients followed over 10 years, Cervera et al. demonstrated an increased risk of thrombotic events, the most common being stroke which was observed in $11.8 \%$ of the cohort [11]. Among SLE patients with and without active disease, both thrombotic events and cognitive impairment have been consistently linked to the presence of a-PL, such as lupus anticoagulant and anticardiolipin antibodies [12]-[15]. Additionally, transverse myelitis was highly associated with the presence of a-PL [16]. Significant correlations between anticardiolipin IgG antibodies and a reduction in psychomotor speed, and between anticardiolipin IgA antibodies and a reduction in conceptual reasoning and executive ability have been found [12]. Conversely the patients who present with typical focal findings, such as motor and cranial nerve deficits, seropositivity for a-PL were not typically associated with cognitive dysfunction and psychiatric disease [17]. While the role of these autoantibodies awaits elucidation, they certainly can act as proxies for disease markers which may aid in the diagnosis of NPSLE.

\section{Anti-Neuronal Antibodies}

The role of anti-neuronal antibodies in the pathogenesis of NPSLE has been appreciated since Bluestein et al. demonstrated that IgG anti-neuronal antibodies were present in much higher concentrations in the cerebrospinal fluid (CSF) from patients with active NPSLE [18]. Of interest, CSF IgG anti-neuronal antibodies were found to be significantly elevated in patients with diffuse NPSLE compared with focal NPSLE [19]. However, the epitopes to which CSF anti-neuronal antibodies were specifically directed have not been fully delineated. As reviewed below, anti-neuronal antibodies that react with SK-N-MC neuroblastoma cell lines may reflect the binding of autoantibodies such as anti-NR2, anti-P, anti-RNP to neuronal cells.

\section{Anti-Sm Antibodies}

It has been pointed out that anti-Sm might be involved in the pathogenesis of NPSLE [20] [21]. Especially, serum anti-Sm was associated with organic brain syndrome, consisting mainly of ACS of diffuse NPSLE [22]. Recently Hirohata et al. showed that CSF anti-Sm levels and Q albumin were significantly higher in ACS than in non-ACS diffuse NPSLE (anxiety disorder, cognitive dysfunction, mood disorder and psychosis) or in focal NPSLE, whereas there was no significant difference in the CSF anti-Sm index among the 3 groups. CSF anti-Sm was significantly correlated with CSF anti-NR2 in NPSLE. They also demonstrated that anti-Sm reacted with neuroblastoma cell lines [23]. This study indicated the possibility that the elevation of CSF anti-Sm and anti-NR2 due to the BBB damage plays a critical role in the pathogenesis of ACS, a severe form of diffuse NPSLE and that anti-Sm are yet other autoantibodies with presumed neural toxicity [23]. The influences of anti-Sm on the function and survival of neurons are currently undetermined and need to be explored in further studies.

\section{Anti-U1-RNP Antibodies}

Sato et al. demonstrated that CSF anti-RNP were associated with NPSLE and CNS manifestations in mixed connective tissue disease as a more specific marker of NPSLE than anti-P or anti-NR2 [24]. However, the elevation of CSF anti-RNP was not specific in ACS or diffuse NPSLE, since CSF anti-RNP was also elevated in patients with focal NPSLE, including aseptic meningitis, headache, demyelinating disorder or movement disorder [24]. Hirohata et al. also showed that CSF anti-RNP antibodies were elevated in focal NPSLE comparably to diffuse NPSLE, confirming the observation in the previous studies [24]. How anti-RNP are involved in the development of NPSLE is currently unknown. Although the possibility of induction of proinflammatory cytokines by anti-RNP was suggested [24], further studies are required to confirm this point.

Additionally anti-RNP may potently induce expression of interferons [25]. The association of anti-RNP with interferons (IFN) in the CSF is described in the section entitled Immune complexes.

\section{Anti-NR2 Glutamate Receptor Binding Antibodies}

$\mathrm{N}$-methyl-D-aspartate (NMDA) receptors are one of the glutamate receptor families and its stimulation has been shown to cause excitatory synaptic transmission in the CNS [26]. DeGiorgio et al. demonstrated that a subset of murine anti-DNA antibodies cross-reacted with a sequence within the NMDA receptor subunit NR2 [27]. Fur- 
thermore, they showed that injection of anti-NR2 (purified antibodies from the sera or CSF from NPSLE patients) into mice brain resulted in apoptosis of the neuronal cells without signs of inflammation [27]. Notably, Kowal et al. demonstrated that mice induced to express anti-NR2 in systemic circulation had no neuronal damage unless breakdown of the BBB took place [28].

Anti-NR2 antibodies were observed in $25 \%$ to $30 \%$ of patients with SLE and might also play a role in cognitive dysfunction and psychiatric disease [29] [30]. We reported a significant relationship between anti-NR2 titers and the presence of NPSLE, especially of complex presentation, such as when focal NPSLE and diffuse NPSLE occur concurrently in a single patient [31]. Accordingly, Hirohata et al. showed that CSF anti-NR2, but not serum anti-NR2, were closely associated with diffuse NPSLE [32].

These studies support the hypothesis that anti-NR2 in the CSF might be strongly associated with the appearance of NPSLE. It has not yet been elucidated whether anti-NR2 are produced outside the CNS and enter the CSF as a result of damage to the BBB or whether they are produced locally. The possibility of damage to the BBB by autoantibodies such as anti-NR2 in SLE patients is reviewed in the section entitled The blood-brain barrier.

\section{Anti-Ribosomal P Protein Antibodies}

Some studies suggested an association between serum anti-P antibodies and diffuse NPSLE of psychosis and depression [19] [33]-[36]. An international meta-analysis of 1537 patients with SLE found negligible value in anti-P titers for the diagnosis of NPSLE or for specific NPSLE manifestations [37]. The potential role of anti-P in the pathogenesis of NPSLE remains controversial.

However, Shoenfeld et al. recently demonstrated that anti-P induced depression in mice when injected intraventricularly [38]. Furthermore Matus et al. demonstrated that anti-P from human lupus serum induced calcium influx and subsequent apoptosis in cortical neurons in rats, which they characterized as a new P-antigen named neuronal surface P antigen (NPSA) [39]. Death of these neurons, found in the hippocampus, amygdala, and certain neo-cortical layers, account for a broad range of potential symptoms, including depression, memory deficits, and cognitive decline. More recently Bravo-Zehnder M. et al. showed that circulating anti-P from NPSLE patients could impair memory when accessing the hippocampus through a permeated BBB in mice and such an effect could occur in the absence of detectable neuronal apoptosis [40]. These results may extend the pathogenic potential of anti-P to cognitive impairment, which is frequent in SLE patients. It is controversial whether anti-P is present in the CSF of SLE patients. Sato et al. reported the lack of anti-P in the CSF of SLE patients [24]. But we demonstrated the presence of anti-P in the CSF of SLE patients and the significant association between the presence of anti-P in the CSF and NPSLE [41]. The studies by Shoenfeld et al. [38] and Matus et al. [39] suggest that the presence of anti-P in the CSF of SLE patients may be closely associated with the pathogenesis of NPSLE, especially diffuse NPSLE.

\section{Anti-Microtubule Associated Protein 2 (MAP-2) Antibodies}

A cellular protein found strictly in neurons and essential to cytoskeletal integrity is MAP-2. The positive frequency of serum anti-MAP-2 antibodies in NPSLE patients was highly significant [42]. Using immunoproteomics, MAP-2B proteins were found to be preferentially recognized by sera from NPSLE patients, which further supported this association between the anti-MAP-2 antibodies and NPSLE [43]. However it has not been elucidated whether CSF anti-MAP-2 antibodies are associated with the pathogenesis of NPSLE. Further studies are necessary to investigate whether serum anti-MAP-2 antibodies enter the BBB and bind to neuronal tissues in the CSF of SLE patient, resulting in the neuronal dysfunction or damage.

\section{The Blood-Brain Barrier}

If autoantibodies such as anti-NR2, anti-Sm, anti-RNP and anti-P are pathogenic in NPSLE, these autoantibodies must be present in CSF of patients with NPSLE. However, it has not been elucidated whether anti-NR2 antibodies are produced outside the CNS and enter the CSF as a result of damage to the BBB or whether they are produced locally. Recently we demonstrated the in vitro activation of human endothelial cells by anti-NR2 (the enhanced production of cytokines such as interleukin-6 (IL-6) and IL-8 and the up-regulated expression of adhesion molecules such as endothelial leukocyte adhesion molecule 1 (ELAM-1), vascular cell adhesion mole- 
cule 1 (VCAM-1), and intercellular adhesion molecule 1 (ICAM-1) through the activation of NF- $\kappa$ B pathway) [44]. Anti-P antibodies have also been shown to bind to endothelial cells and activate endothelial cells.

Our findings also support the possibility that anti-NR2, anti-P and other autoantibodies are produced outside the CNS and enter the CSF as a result of the inflammation of cerebral endothelial cells that is caused by at least anti-NR2 and anti-P antibodies.

Further studies are required to elucidate whether anti-NR2 antibodies in the CSF bind to neuronal tissue and cause neuronal dysfunction or damage and whether this causes the cognitive disturbance and memory loss commonly observed in SLE patients.

\section{Anti-Endothelial Cell Antibodies}

Conti et al. found that $64.7 \%$ of NPSLE patient's sera were positive for anti-endothelial cell antibodies (AECA), as compared to only $29.4 \%$ of non-NPSLE patients [45].

AECA have been previously characterized as inducers of increased endothelial cellular adhesion molecules, including ELAM-1, ICAM-1, and VCAM-1. We stimulated human umbilical endothelial cells with monoclonal antibodies targeted toward thrombomodulin, a proposed antigenic substrate of some AECA in SLE patients [46], and found increased endothelial production of IL-6 and IL-8 mediated through the NF- $\kappa$ B pathway [47]. More recently, we demonstrated that anti-NR2 recognized antigenic targets on human umbilical endothelial cells, and induced the increased production of IL-6 and IL-8 from human umbilical endothelial cells and the up-regulated expression of adhesion molecules on human umbilical endothelial cells without IL- $1 \beta$ co-treatment [44] as described in the section entitled The blood-brain barrier.

Collectively, AECA may consist of anti-NR2, anti-P and anti-PL antibodies and induce endothelial activation, which is pivotal in many inflammatory processes, while in the brain these antibodies play an important role in disruption of the BBB [48].

\section{Immune Complexes}

Since IFN- $\alpha$ was reported to be detected in the CSF from five out of six NPSLE patients and in the microglia and neurons following autopsy analysis of a patient who died from diffuse NPSLE [49], IFN- $\alpha$ has been suggested to be directly implicated as a causative factor in NPSLE. Furthermore, IC containing nucleic acid released by necrotic or late apoptic cells and IgG derived from sera with SLE patients were reported to induce the IFN- $\alpha$ production in plasmacytoid dendritic cells [50]. These IC were endocytosed by Fc $\gamma$ receptors on plasmacytoid dendritic cells and induced IFN production by nucleic acid activation of toll-like receptors [51] [52].

Santer et al. presented the data showing whether NPSLE patient serum and/or CSF contain abnormally high IFN- $\alpha$-inducing activity using a bioassay containing plasmacytoid dendritic cells and a source of antigen [25]. They found that NPSLE CSF induced significantly higher IFN- $\alpha$ compared with CSF from patients with multiple sclerosis or other autoimmune disease controls [25]. In addition to IFN- $\alpha$, IC formed by CSF autoantibodies produced significantly increased levels of IFN- $\gamma$-inducible protein 10 (IP-10/CXCL), IL-8, and monocyte chemotactic protein 1 (MCP-1)/CCL2, all of which have been reported to be elevated in the CSF from NPSLE patients [25]. The generation of IFN in patients with SLE was caused, at least partially, by autoantibodies that bind to RNP particles released from dead and dying cells [25]. IFN-inducing activity in the CSF was correlated with serum anti-U1 RNP antibodies but not with other known antinuclear antibodies [25]. Therefore, anti-U1 RNP antibodies and their IC in CSF may have pathogenic roles in NPSLE.

We reported that CSF IgG-IC levels in NPSLE patients were significantly higher than non-NPSLE patients [53]. But the intrathecal IgG-IC was not associated with the intrathecal production of any cytokines and chemokines including IFN- $\alpha$ [53]. It is unclear whether the same findings as these in vitro results appear intrathecally in SLE patients and are associated with the pathogenesis of NPSLE.

\section{Cytokines and Chemokines}

Increased levels of cytokines and chemokines have been reported in the CSF of NPSLE patients and some reports have shown cytokines and chemokines such as IL-6, IL-1, IL-8, IL-10, tumor necrosis factor- $\alpha$ (TNF- $\alpha$, IFN- $\alpha$, MCP-1, IFN- $\gamma$ inducible protein-10 (IP-10)/CXCL10 and regulated on activation normal T cell expressed 
and secreted (RANTES) to be elevated intrathecally, thereby allowing these cytokines and chemokines to be used as diagnostic tools [54]-[58].

Recently we showed that the concentrations of IL-6, IL-8, IP-10, MCP-1 and granulocyte-colony stimulating factor (G-CSF)were higher in the CSF than those in the sera, respectively, while the concentrations of IL-1, IL10 , TNF- $\alpha$ RANTES and IFN- $\alpha$ in the CSF were very much lower than those in the sera, respectivelyin NPSLE patients [59]. These results suggested that the intrathecal concentrations of cytokines and chemokines are not influenced by the serum concentrations, indicating that the production of IL-6, IL-8, IP-10, MCP-1 and G-CSF might take place in the CNS [59]. These increased CSF cytokines and chemokines (IL-6, IL-8, IP-10, MCP-1 and G-CSF) may be associated with the pathogenesis and appearance of NPSLE. The measurement of these cytokines and chemokines, especially IL-6 might be useful for the diagnosis of central NPSLE.

As reviewed in the section entitled The blood-brain barrier, recently we demonstrated the in vitro activation of human endothelial cells by anti-NR2 (the enhanced production of cytokines such as IL-6 and IL-8 and the upregulated expression of adhesion molecules such as ELAM-1, ICAM-1 and VCAM-1) through the activation of NF-kB pathway [44]. IL-6, IL-8, IP-10, MCP-1 and G-CSF are produced by endothelial cells. Significantly increased IL-6, IL-8, IP-10, MCP-1 and G-CSF levels in the CSF than in the sera in NPSLE patients may be derived from endothelial cells of the BBB.

Although CSF IL-6, IL-8, IP-10 and MCP-1 levels have previously been reported to be higher in patients with NPSLE than in non-NPSLE patients [54]-[58], the increased levels of CSF G-CSF in patients with NPSLE have been not yet shown. Firstly we observed the significant increased levels of CSF G-CSF compared with serum simultaneous levels in patients with NPSLE and the significant increased levels of CSF G-CSF in patients with NPSLE compared with patients with non-NPSLE [59]. Recently CSF G-CSF levels have been reported to be significantly higher in patients with neuromyelitisoptica than in patients with other non-inflammatory neurological diseases [60]. Besides the function of hematopoietic effect, G-CSF can also act as neurotrophic factor, induce neuorogenesis and to counteract apoptosis. These properties play a major role in the development of treatments of neurological diseases such as cerebral ischemia [61] [62]. In patients with central NPSLE, G-CSF might act to treat the damaged CNS intrathecally.

Intrathecal production of these cytokines and chemokines by neuronal or glial cells may also take place. These cytokines and chemokines may increase the permeability of the BBB, thus providing access for circulating autoantibodies and leukocytes to the CNS. It is conceivable that both the degree of the BBB dysfunction and the type and titer of autoantibodies might be the determining factors in the development of certain diffuse NPSLE, such as psychosis and ACS.

\section{Two Kinds or More Than Two Kinds of Autoantibodies}

Since a number of autoantibodies have been reported to react to neurons, including anti-Sm [23], anti-P [63], anti-Ro antibodies [64], as well as some anti-cardiolipin [8] and anti-NR2 antibodies [27] [28] [32], it is possible that the patterns of expression of several antibodies or their combination in CSF may be different between diffuse and focal NPSLE. Moreover, Hirohata et al. suggested the possibility that anti-P, anti-Ro antibodies and some anti-cardiolipin antibodies are also involved in the development of ACS in combination with anti-Sm and anti-NR2 antibodies [23].

\section{Conclusions}

As reviewed above, many aforementioned autoantibodies in the CSF have reported to be significantly associated with the appearance of NPSLE and these autoantibodies might have important roles in the pathogenesis of NPSLE. Intrathecal IC which consists of aforementioned autoantibodies and their epitopes may bind to neuronal cells to promote inflammation with complements, resulting in damage to the brain and changes in the neuronal function through the activation of signaling pathways such as NF- $\kappa \mathrm{B}$ and caspase. This activation of signaling pathways promotes the release of cytokines and chemokines such as IL-6 from neuronal cells resulting in the pathology of NPSLE. This pathology in the CNS might reflect the elevation of autoantibody titers, presence of IC, and the increased cytokines and chemokines in the CSF of NPSLE patients.

These pathogenic autoantibodies have to be present intrathecally to work as triggering factors of inflammation in CSF. However, whether autoantibodies are produced intrathecally or enter the CSF through the breach of 
BBB remains unclear, thus far.

A growing body of evidence has shown that NPSLE is often associated with the presence of autoantibodies within the CNS, making the question of how these autoantibodies gain entry into this anatomically privileged space increasingly important. One possibility is de novo production of these autoantibodies in CSF, as opposed to entry from systemic circulation. If that is the case, these autoantibodies must be produced by plasma cells differentiated from activated B cells. In the case of multiple sclerosis (MS), B-cell counts and the B-cell attractant chemokine CXCL13 are increased in the CSF of MS patients [65]. Thus far, there is no definitive evidence suggesting that autoantibodies within the CNS are produced by B cells intrathecally. Therefore, B-cell counts and CXCL13 levels in CSF should be evaluated in patients with NPSLE to draw a clear picture that how some autoantibodies are elevated in the CSF of NPSLE patients. The other possibility is permeability changes in the BBB allowing the entry of pathogenic autoantibodies from systemic circulation to CSF, following the direct interaction with neurons. There is some evidence in the literature supporting this possibility. The presence of the elevation of Q albumin index in NPSLE patients might support entry of circulating autoantibodies across the BBB. Evidence points to entry of autoantibodies across the BBB, with entry into different brain regions and specific autoantibody subtypes potentially associated with the variable phenotypes found in both murine experimental models and NPSLE patients. There is strong support for the roles of AECA which include anti-NR2, anti-P and anti-PL antibodies, complement components, and environmental mediators in increasing permeability across the BBB, though in each of these cases, cytokines and chemokines have an essential role as well. Finally we propose a hypothesis by which IgG anti-NR2 antibodies activate the NF-kB pathway, resulting in the pathogenesis of NPSLE (Figure 1) [44].

Further studies are required to elucidate the role of pathogenic autoantibodies in the pathogenesis of NPSLE.

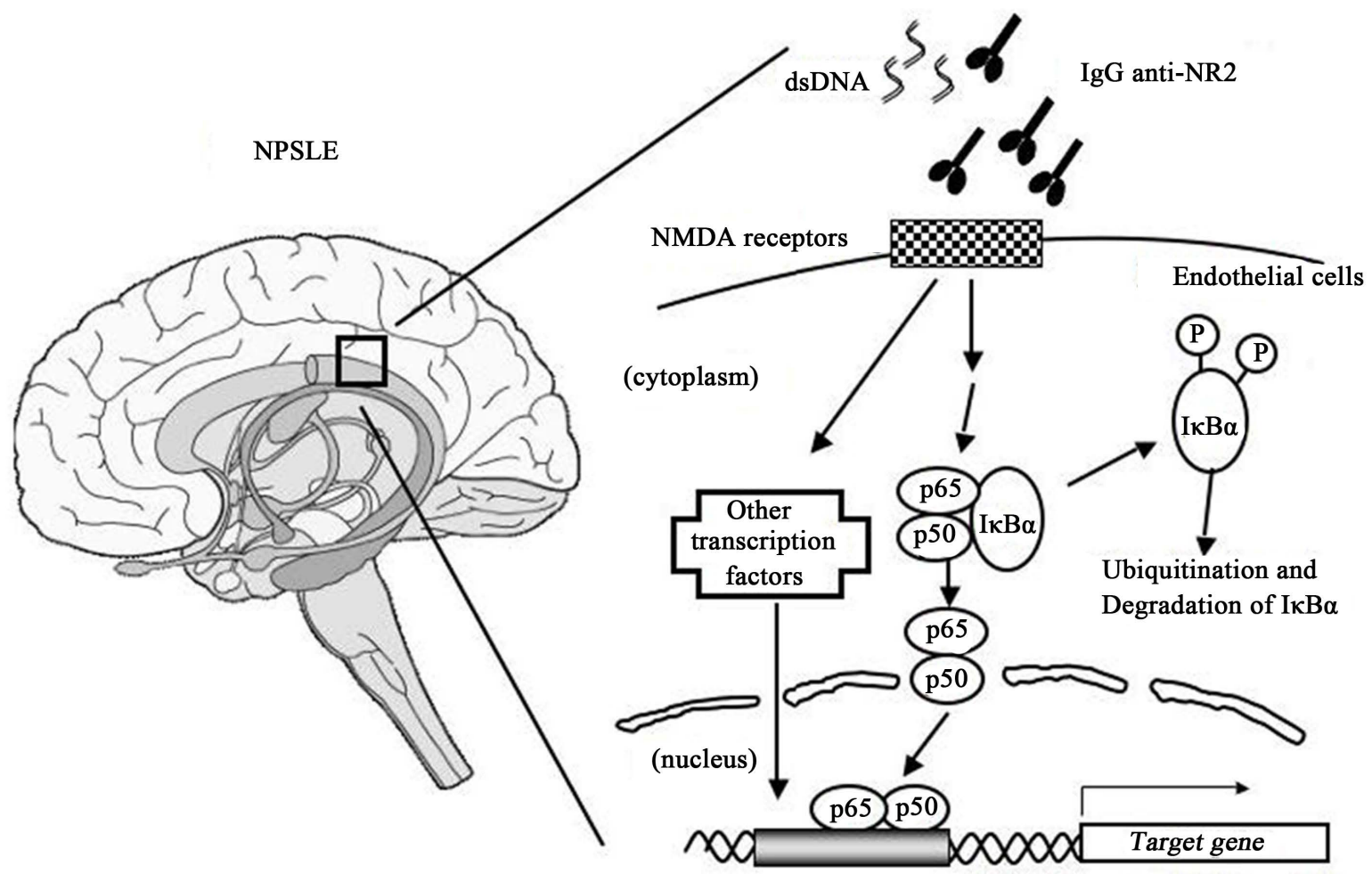

Cytokines and Chemokines

Figure 1. Schematic representation of the mechanism by which IgG anti-NR2 glutamate receptor antibodies activate the NF- $\kappa$ B pathway, resulting in the pathogenesis of neuropsychiatric systemic lupus erythematosus (NPSLE). IgG anti-NR2 glutamate receptor antibodies that are cross-reactive with anti-double-stranded DNA (anti-dsDNA) antibodies might bind to endothelial cells (ECs) through N-methyl-D-aspartate (NMDA) receptor subunits NR2a and NR2b, resulting in activation of $\mathrm{NF}-\kappa \mathrm{B}$ signaling by ECs, promoting the pathogenesis of NPSLE. Encircled $\mathrm{p}$ attached to $\mathrm{I} \kappa \mathrm{B} \alpha$ indicates phosphorylated $\mathrm{I} \kappa \mathrm{B} \alpha$. 


\section{Conflicts}

Authors have no conflict of interest to declare.

\section{References}

[1] Belmont, H.M., Abramson, S.B. and Lie, J.T. (1996) Pathology and Pathogenesis of Vascular Injury in Systemic Lupus Erythematosus. Interactions of Inflammatory Cells and Activated Endothelium. Arthritis Rheumatology, 39, 9-22. http://dx.doi.org/10.1002/art.1780390103

[2] Hanly, J.G., Walsh, N.M. and Sangalang, V. (1992) Brain Pathology in Systemic Lupus Erythematosus. Journal of Rheumatology, 19, 732-741.

[3] Abbott, N.J., Mendonca, L.L. and Dolman, D.E. (2003) The Blood-Brain Barrier in Systemic Lupus Erythematosus. Lupus, 12, 908-915. http://dx.doi.org/10.1191/0961203303lu501oa

[4] Devreese, K.M. (2012) Standardization of Antiphospholipid Antibody Assays. Where Do We Stand? Lupus, 21, 718-721. http://dx.doi.org/10.1177/0961203312439335

[5] Kent, M., Alvarez, F., Vogt, E., Fyffe, R., Ng, A.K. and Rote, N. (1997) Monoclonal Antiphosphatidylserine Antibodies React Directly with Feline and Murine Central Nervous System. Journal of Rheumatology, 24, 1725-1733.

[6] Shoenfeld, Y., Nahum, A., Korczyn, A.D., Dano, M., Rabinowitz, R., Beilin, O., et al. (2003) Neuronal-Binding Antibodies from Patients with Antiphospholipid Syndrome Induce Cognitive Deficits Following Intrathecal Passive Transfer. Lupus, 12, 436-442. http://dx.doi.org/10.1191/0961203303lu409oa

[7] Caronti, B., Calderaro, C., Alessandri, C., Conti, F., Tinghino, R., Pini, C., et al. (1998) Serum Anti-Beta2-Glycoprotein I Antibodies from Patients with Antiphospholipid Antibody Syndrome Bind Central Nervous System Cells. Journal of Autoimmunity, 11, 425-429. http://dx.doi.org/10.1006/jaut.1998.0214

[8] Caronti, B., Pittoni, V., Palladini, G. and Valesini, G. (1998) Anti-Beta 2-Glycoprotein I Antibodies Bind to Central Nervous System. Journal of Neuroscience, 156, 211-219.

[9] Denburg, S.D., Carbotte, R.M. and Denburg, J.A. (1987) Cognitive Impairment in Systemic Lupus Erythematosus: A Neuropsychological Study of Individual and Group Deficits. Journal of Clinical and Experimental Neuropsychology, 9, 323-339. http://dx.doi.org/10.1080/01688638708405054

[10] Hanly, J.G., Robichaud, J. and Fisk, J.D. (2006) Anti-NR2 Glutamate Receptor Antibodies and Cognitive Function in Systemic Lupus Erythematosus. Journal of Rheumatology, 33, 1553-1558.

[11] Cervera, R., Khamashta, M.A., Font, J., Sebastiani, G.D., Gil, A., Lavilla, P., et al. (2003) Morbidity and Mortality in Systemic Lupus Erythematosus during a 10-Year Period: A Comparison of Early and Late Manifestations in a Cohort of 1,000 Patients. Medicine, 82, 299-308. http://dx.doi.org/10.1097/01.md.0000091181.93122.55

[12] Hanly, J.G., Hong, C., Smith, S. and Fisk, J.D. (1999) A Prospective Analysis of Cognitive Function and Anticardiolipin Antibodies in Systemic Lupus Erythematosus. Arthritis \& Rheumatism, 42, 728-734. http://dx.doi.org/10.1002/1529-0131(199904)42:4<728::AID-ANR16>3.0.CO;2-O

[13] Menon, S., Jameson-Shortall, E., Newman, S.P., Hall-Craggs, M.R., Chinn, R. and Isenberg, D.A. (1999) A Longitudinal Study of Anticardiolipin Antibody Levels and Cognitive Functioning in Systemic Lupus Erythematosus. Arthritis \& Rheumatism, 42, 735-741. http://dx.doi.org/10.1002/1529-0131(199904)42:4<735::AID-ANR17>3.0.CO;2-L

[14] Shah, N.M., Khamashta, M.A., Atsumi, T. and Hughes, G.R. (1998) Outcome of Patients with Anticardiolipin Antibodies: A 10 Year Follow-Up of 52 Patients. Lupus, 7, 3-6. http://dx.doi.org/10.1191/096120398678919624

[15] Toubi, E., Khamashta, M.A., Panarra, A. and Hughes, G.R. (1995) Association of Antiphospholipid Antibodies with Central Nervous System Disease in Systemic Lupus Erythematosus. The American Journal of Medicine, 99, 397-401. http://dx.doi.org/10.1016/S0002-9343(99)80188-0

[16] Rodriguez, A.S., Oterino, J.A. and Hernandez, P.A. (1998) Lupus Myelitis. Archives of Internal Medicine, 158, 22672268. http://dx.doi.org/10.1001/archinte.158.20.2267

[17] Borowoy, A.M., Pope, J.E., Silverman, E., Fortin, P.R., Pineau, C., Smith, C.D., et al. (2012) Neuropsychiatric Lupus: The Prevalence and Autoantibody Associations Depend on the Definition: Results from the 1000 Faces of Lupus Cohort. Seminars in Arthritis and Rheumatism, 42, 179-185. http://dx.doi.org/10.1016/j.semarthrit.2012.03.011

[18] Bluestein, H.G., Williams, G.W. and Steinberg, A.D. (1981) Cerebrospinal Fluid Antibodies to Neuronal Cells: Association with Neuropsychiatric Manifestations of Systemic Lupus Erythematosus. The American Journal of Medicine, 70, 240-246. http://dx.doi.org/10.1016/0002-9343(81)90756-7

[19] Isshi, K. and Hirohata, S. (1998) Differential Roles of the Anti-Ribosomal P Antibody and Antineuronal Antibody in the Pathogenesis of Central Nervous System Involvement in Systemic Lupus Erythematosus. Arthritis \& Rheumatism, 41, 1819-1827. http://dx.doi.org/10.1002/1529-0131(199810)41:10<1819::AID-ART14>3.0.CO;2-Y 
[20] Winfield, J.B., Brunner, C.M. and Koffler, D. (1978) Serologic Studies in Patients with Systemic Lupus Erythematosus and Central Nervous System Dysfunction. Arthritis \& Rheumatism, 21, 289-294. http://dx.doi.org/10.1002/art.1780210301

[21] Yasuma, M., Takasaki, Y., Matsumoto, K., Kodama, A., Hashimoto, H. and Hirose, S. (1990) Clinical Significance of IgG Anti-Sm Antibodies in Patients with Systemic Lupus Erythematosus. The Journal of Rheumatology, 17, 469-475.

[22] Hirohata, S. and Kosaka, M. (1994) Association of Anti-Sm Antibodies with Organic Brain Syndrome Secondary to Systemic Lupus Erythematosus. The Lancet, 343, 796. http://dx.doi.org/10.1016/S0140-6736(94)91875-9

[23] Hirohata, S., Sakuma, Y., Yanagida, T. and Yoshio, T. (2014) Association of Cerebrospinal Fluid Anti-Sm Antibodies with Acute Confusional State in Systemic Lupus Erythematosus. Arthritis Research \& Therapy, 16, 450. http://dx.doi.org/10.1186/s13075-014-0450-z

[24] Sato, T., Fujii, T., Yokoyama, T., Fujita, Y., Imura, Y., Yukawa, N., et al. (2010) Anti-U1 RNP Antibodies in Cerebrospinal Fluid Are Associated with Central Neuropsychiatric Manifestations in Systemic Lupus Erythematosus and Mixed Connective Tissue Disease. Arthritis Research \& Therapy, 62, 3730-3740. http://dx.doi.org/10.1002/art.27700

[25] Santer, D.M., Yoshio, T., Minota, S., Moller, T. and Elkon, K.B. (2009) Potent Induction of IFN- $\alpha$ and Chemokines by Autoantibodies in the Cerebrospinal Fluid of Patients with Neuropsychiatric Lupus. The Journal of Immunology, 182, 1192-1201. http://dx.doi.org/10.4049/jimmunol.182.2.1192

[26] Furukawa, H., Singh, S.K., Mancusso, R. and Gouaux, E. (2005) Subunit Arrangement and Function in NMDA Receptors. Nature, 438, 185-192. http://dx.doi.org/10.1038/nature04089

[27] De Giorgio, L.A., Konstantinov, K.N., Lee, S.C., Hardin, J.A., Volpe, B.T. and Diamond, B. (2001) A Subset of Lupus Anti-DNA Antibodies Cross-Reacts with the NR2 Glutamate Receptor in Systemic Lupus Erythematosus. Nature Medicine, 7, 1189-1193. http://dx.doi.org/10.1038/nm1101-1189

[28] Kowal, C., De Giorgio, L.A., Nakaoka, T., Hetherington, H., Huerta, P.T., Diamond, B., Volpe, B.T., et al. (2004) Cognition and Immunity: Antibody Impairs Memory. Immunity, 21, 179-188. http://dx.doi.org/10.1016/j.immuni.2004.07.011

[29] Husebye, E.S., Sthoeger, Z.M., Dayan, M., Zinger, H., Elbirt, D., Levite, M., et al. (2005) Autoantibodies to a NR2A Peptide of the Glutamate/NMDA Receptor in Sera of Patients with Systemic Lupus Erythematosus. Annals of the Rheumatic Diseases, 64, 1210-1213. http://dx.doi.org/10.1136/ard.2004.029280

[30] Omdal, R., Brokstad, K., Waterloo, K., Koldingsnes, W., Jonsson, R. and Mellgren, S.I. (2005) Neuropsychiatric Disturbances in SLE Are Associated with Antibodies Against NMDA Receptors. European Journal of Neurology, 12, 392-398. http://dx.doi.org/10.1111/j.1468-1331.2004.00976.x

[31] Yoshio, T., Onda, K., Nara, H. and Minota, S. (2006) Association of IgG Anti-NR2 Glutamate Receptor Antibodies in Cerebrospinal Fluid with Neuropsychiatric Systemic Lupus Erythematosus. Arthritis \& Rheumatism, 54, 675-678. http://dx.doi.org/10.1002/art.21547

[32] Arinuma, Y., Yanagida, T. and Hirohata, S. (2008) Association of Cerebrospinal Fluid Anti-NR2 Glutamate Receptor Antibodies with Diffuse Neuropsychiatric Systemic Lupus Erythematosus. Arthritis \& Rheumatism, 58, 1130-1135. http://dx.doi.org/10.1002/art.23399

[33] Nojima, Y., Minota, S., Yamda, A., Takaku, F., Aotsuka, S. and Yokohari, R. (1992) Correlation of Antibodies to Ribosomal P Protein with Psychosis in Patients with Systemic Lupus Erythematosus. Annals of the Rheumatic Diseases, 51, 1053-1055. http://dx.doi.org/10.1136/ard.51.9.1053

[34] Schneebaum, A.B., Singleton, J.D., West, S.G., Blodgett, J.K., Allen, L.G., Cheronis, J.C., et al. (1991) Association of Psychiatric Manifestations with Antibodies to Ribosomal P Proteins in Systemic Lupus Erythematosus. The American Journal of Medicine, 90, 54-62. http://dx.doi.org/10.1016/0002-9343(91)90506-S

[35] Teh, L.S., Bedwell, A.E., Isenberg, D.A., Gordon, C., Emery, P., Charles, P.J., et al. (1992) Antibodies to Protein P in Systemic Lupus Erythematosus. Annals of the Rheumatic Diseases, 51, 489-494. http://dx.doi.org/10.1136/ard.51.4.489

[36] Georgescu, L., Mevorach, D., Arnett, F.C., Reveille, J.D. and Elkon, K.B. (1997) Anti-P Antibodies and Neuropsychiatric Lupus Erythematosus. Annals of the New York Academy of Sciences, 823, 263-269. http://dx.doi.org/10.1111/j.1749-6632.1997.tb48399.x

[37] Karassa, F.B., Afeltra, A., Ambrozic, A., Chang, D.M., De Keyser, F., Doria, A., et al. (2006) Accuracy of Anti-Ribosomal P Protein Antibody Testing for the Diagnosis of Neuropsychiatric Systemic Lupus Erythematosus: An International Meta-Analysis. Arthritis \& Rheumatism, 54, 312-314. http://dx.doi.org/10.1002/art.21539

[38] Katzav, A., Solodeev, I., Brodsky, O., Chapman, J., Pick, C.G., Blank, M., et al. (2007) Induction of Autoimmune Depression in Mice by Anti-Ribosomal P Antibodies via the Limbicsystem. Arthritis \& Rheumatism, 56, 938-948. http://dx.doi.org/10.1002/art.22419

[39] Matus, S., Burgos, P.V., Bravo-Zehnder, M., Kraft, R., Porras, O.H., Farías, P., et al. (2007) Antiribosomal-P Autoan- 
tibodies from Psychiatric Lupus Target a Novel Neuronal Surface Protein Causing Calcium Influx and Apoptosis. The Journal of Experimental Medicine, 204, 3221-3234. http://dx.doi.org/10.1084/jem.20071285

[40] Bravo-Zehnder, M., Toledo, E.M., Segovia-Miranda, F., Serrano, F.G., Benito, M.J., Metz, C., et al. (2015) Anti-Ribosomal P Protein Autoantibodies from Neuropsychiatric Lupus Impair Memory. Arthritis \& Rheumatology, 67, $204-$ 214. http://dx.doi.org/10.1002/art.38900

[41] Yoshio, T., Hirata, D., Onda, K., Nara, H. and Minota, S. (2005) Antiribosomal P Protein Antibodies in Cerebrospinal Fluid Are Associated with Neuropsychiatric Systemic Lupus Erythematosus. The Journal of Rheumatology, 32, 34-39.

[42] Williams Jr., R.C., Sugiura, K. and Tan, E.M. (2004) Antibodies to Microtubule-Associated Protein 2 in Patients with Neuropsychiatric Systemic Lupus Erythematosus. Arthritis \& Rheumatism, 50, 1239-1247. http://dx.doi.org/10.1002/art.20156

[43] Lefranc, D., Launay, D., Dubucquoi, S., de Seze, J., Dussart, P., Vermersch, M., et al. (2007) Characterization of Discriminant Human Brain Antigenic Targets in Neuropsychiatric Systemic Lupus Erythematosus Using an Immunoproteomic Approach. Arthritis \& Rheumatism, 56, 3420-3432. http://dx.doi.org/10.1002/art.22863

[44] Yoshio, T., Okamoto, H., Hirohata, S. and Minota, S. (2013) IgG Anti-NR2 Glutamate Receptor Autoantibodies from Patients with Systemic Lupus Erythematosus Activate Endothelial Cells. Arthritis \& Rheumatism, 65, 457-463. http://dx.doi.org/10.1002/art.37745

[45] Conti, F., Alessandri, C., Bompane, D., Bombardieri, M., Spinelli, F.R., Rusconi, A.C., et al. (2004) Autoantibody Profile in Systemic Lupus Erythematosus with Psychiatric Manifestations: A Role for Anti-Endothelial-Cell Antibodies. Arthritis Research \& Therapy, 6, R366-R372. http://dx.doi.org/10.1186/ar1198

[46] Oosting, J.D., Preissner, K.T., Derksen, R.H. and de Groot, P.G. (1993) Autoantibodies Directed Against the Epidermal Growth Factor-Like Domains of Thrombomodulin Inhibit Protein C Activation in Vitro. British Journal of Haematology, 85, 761-768. http://dx.doi.org/10.1111/j.1365-2141.1993.tb03220.x

[47] Nara, H., Okamoto, H., Minota, S. and Yoshio, T. (2006) Mouse Monoclonal Anti-Human Thrombomodulin Antibodies Bind to and Activate Endothelial Cells Through NF- $\kappa$ B Activation in Vitro. Arthritis \& Rheumatism, 54, 16291637. http://dx.doi.org/10.1002/art.21797

[48] Wong, D., Dorovini-Zis, K. and Vincent, S.R. (2004) Cytokines, Nitric Oxide, and cGMP Modulate the Permeability of an in Vitro Model of the Human Blood-Brain Barrier. Experimental Neurology, 190, 446-455. http://dx.doi.org/10.1016/j.expneurol.2004.08.008

[49] Shiozawa, S., Kuoroki, Y., Kim, M., Hirohata, S. and Ogino, T. (1992) Interferon-Alpha in Lupus Psychosis. Arthritis \& Rheumatism, 35, 417-422. http://dx.doi.org/10.1002/art.1780350410

[50] Lovgren, T., Eloranta, M.L., Bave, U., Alm, G.V. and Ronnblom, L. (2004) Induction of Interferon-Production in Plasmacytoid Dendritic Cells by Immune Complexes Containing Nucleic Acid Released by Necrotic or Late Apoptotic Cells and Lupus IgG. Arthritis \& Rheumatism, 50, 1861-1872. http://dx.doi.org/10.1002/art.20254

[51] Martin, D.A. and Elkon, K.B. (2005) Autoantibodies Make a U-Turn: The Toll Hypothesis for Autoantibody Specificity. The Journal of Experimental Medicine, 202, 1465-1469. http://dx.doi.org/10.1084/jem.20052228

[52] Marshak-Rothstein, A. and Rifkin, I.R. (2007) Immunologically Active Autoantigens: The Role of Toll-Like Receptors in the Development of Chronic Inflammatory Disease. Annual Review of Immunology, 25, 419-441. http://dx.doi.org/10.1146/annurev.immunol.22.012703.104514

[53] Yoshio, T., Okamoto, H., Kurasawa, K., Dei, Y., Hirohata, S. and Minota, S. (2013) The Presence of IgG-Immune Complexes in the Cerebrospinal Fluids Is Associated with Central Neurocychiatricmanifestatin but Not with Intrathecal Production of Proimmflammatory Cytokines/Chemokines Such as Interferon- $\alpha$ in Systemic Lupus Erythematosus. Arthritis \& Rheumatism, 65, S1593.

[54] Trysberg, E., Carlsten, H. and Tarkowski, A. (2000) Intrathecal Cytokines in Systemic Lupus Erythematosus with Central Nervous System Involvement. Lupus, 9, 498-503. http://dx.doi.org/10.1177/096120330000900704

[55] Svenungsson, E., Andersson, M., Brundin, L.,van Vollenhoven, R., Khademi, M., Tarkowski, A., et al. (2001) Increased Levels of Proinflammatory Cytokines and Nitric Oxide Metabolites in Neuropsychiatric Lupus Erythematosus. Annals of the Rheumatic Diseases, 60, 372-379. http://dx.doi.org/10.1136/ard.60.4.372

[56] Iikuni, N., Okamoto, H., Yoshio, T., Sato, E., Kamitsuji, S., Iwamoto, T., et al. (2006) Raised Monocyte Chemotactic Protein-1 (MCP-1)/CCL2 in Cerebrospinal Fluid of Patients with Neuropsychiatric Lupus. Annals of the Rheumatic Diseases, 65, 253-256. http://dx.doi.org/10.1136/ard.2005.041640

[57] Okamoto, H., Iikuni, N., Kamitsuji, S., Yoshio, T., Minota, S. and Kamatani, N. (2006) IP-10/MCP-1 Ratio in CSF Is an Useful Diagnostic Marker of Neuropsychiatric Lupus Patients. Rheumatology, 45, 232-234. http://dx.doi.org/10.1093/rheumatology/kei233

[58] Okamoto, H., Katsumata, Y., Nishimura, K. and Kamatani, N. (2004) Interferon-Inducible Protein 10/CXCL10 Is Increased in the Cerebrospinal Fluid of Patients with Central Nervous System Lupus. Arthritis \& Rheumatism, 50, 3731- 
3732. http://dx.doi.org/10.1002/art.20598

[59] Yoshio, T., Okamoto, H., Kurasawa, K., Dei, Y., Hirohata, S. and Minota, S. (2011) The Intrathecal Production of IL-6, IL-8, IP-10, MCP-1 and G-CSF Increases More Highly Than the Serum Production in Patients with Central Neuropsychiatric Lupus Erythematosus. Arthritis \& Rheumatism, 63, S1711.

[60] Matsushita, T., Tateishi, T., Isobe, N., Yonekawa, T., Yamasaki, R., Matsuse, D., et al. (2013) Characteristic Cerebrospinal Fluid Cytokine/Chemokine Profiles in Neuromyelitisoptica, Relapsing Remitting or Primary Progressive Multiple Sclerosis. PLoS ONE, 8, e61835. http://dx.doi.org/10.1371/journal.pone.0061835

[61] Schneider, A., Kruger, C., Steigleder, T., Weber, D., Pitzer, C., Laage, R., et al. (2005) The Hematopoietic Factor G-CSF Is a Neuronal Ligand That Counteracts Programmed Cell Death and Drives Neurogenesis. The Journal of Clinical Investigation, 115, 2083-2098. http://dx.doi.org/10.1172/JCI23559

[62] Pitzer, C., Krüger, C., Plaas, C., Kirsch, F., Dittgen, T., Müller, R., et al. (2008) Granulocyte-Colony Stimulating Factor Improves Outcome in a Mouse Model of Amyotrophic Lateral Sclerosis. Brain, 131, 3335-3347. http://dx.doi.org/10.1093/brain/awn243

[63] Hirohata, S., Arinuma, Y., Takayama, M. and Yoshio, T. (2007) Association of Cerebrospinal Fluid Anti-Ribosomal P Protein Antibodies with Diffuse Psychiatric/Neuropsychological Syndromes in Systemic Lupus Erythematosus. Arthritis Research \& Therapy, 9, R44. http://dx.doi.org/10.1186/ar2184

[64] Fancellu, R., Pareyson, D., Corsini, E., Salsano, E., Laurà, M., Bernardi, G., et al. (2009) Immunological Reactivity against Neuronal and Non-Neuronal Antigens in Sporadic Adult-Onset Cerebellar Ataxia. European Neurology, 62, 356-361. http://dx.doi.org/10.1159/000242010

[65] Disanto, G., Morahan, J.M., Barnett, M.H., Giovannoni, G. and Ramagopalan, S.V. (2012) The Evidence for a Role of B Cells in Multiple Sclerosis. Neurology, 78, 823-832. http://dx.doi.org/10.1212/WNL.0b013e318249f6f0 